\title{
Exenatide at mealtimes in type 1 diabetes-no MAG1C with exenatide, or with other glucagon-like peptide-1 receptor agonists
}

\author{
Sheila A. Doggrell \\ Faculty of Health, Queensland University of Technology, Brisbane, QLD 4002, Australia \\ Correspondence to: Sheila A. Doggrell. Faculty of Health, Queensland University of Technology, Brisbane, QLD 4002, Australia. \\ Email: sheila.doggrell@qut.edu.au. \\ Comment on: Johansen NJ, Dejgaard TF, Lund A, et al. Efficacy and safety of meal-time administration of short-acting exenatide for glycaemic \\ control in type 1 diabetes (MAG1C): a randomised, double-blind, placebo-controlled trial. Lancet Diabetes Endocrinol 2020;8:313-24.
}

Submitted May 04, 2020. Accepted for publication Jun 11, 2020.

doi: 10.21037/atm-20-3702

View this article at: http://dx.doi.org/10.21037/atm-20-3702

In type 1 and 2 diabetes mellitus, reduced insulin secretion, insulin action, or the combination lead to chronic hyperglycaemia, and this is characterised by failure of the eyes, kidneys, nerves, and cardiovascular system. In type 1 diabetes mellitus (T1DM), the lack of insulin secretion is due to cellular-medicated autoimmune destruction of the pancreatic $\beta$-cells. This is accompanied by an increased secretion of glucagon from the pancreatic $\alpha$-cells which releases glucose from the liver. Lowering this could reduce hyperglycaemia (1). With a reduction or no insulin secretion in T1DM, the logical treatment is to replace the insulin. However, despite insulin replacement therapy in T1DM, the incidence and prevalence of cardiovascular disease is still raised, and this includes myocardial infarction, coronary revascularisation, death from coronary heart disease or stroke, non-fatal stroke, and peripheral vascular disease (2). Damage to the kidney (2), eye (3) and peripheral nervous system (4) in T1DM is also associated with the increase in cardiovascular/microvascular disease.

Hypoglycaemia episodes limit the use of insulin replacement therapy in T1DM. These episodes are more common with intense/higher than with conventional/lower doses of insulin. However, if the lower doses of insulin are used to prevent hypoglycaemia there is poor glycaemic control (5). Weight gain is another factor limiting insulin replacement therapy use in T1DM. This is increasingly being observed and may increase cardiovascular risk (6).

Consequently, the search for better treatments for T1DM continues. Adjunct therapy to insulin is one approach to improve glycaemic control and prevent the use of high doses of insulin prior to meals, which are responsible for the excessive hypoglycaemia. Adjunct therapy is used to reduce insulin dose and the consequent weight gain. In T1DM, a group of drugs being tested as adjuncts to insulin are the glucagon-like peptide-1 receptor (GLP-1R) agonists such as exenatide. With a meal, the intestinal hormone glucagon-like peptide 1 (GLP-1) is secreted, which in turn increases glucose-mediated insulin secretion and suppresses glucagon secretion from the pancreatic $\alpha$-cells to reduce glucose levels. In addition, GLP-1 inhibits gastric emptying and reduces appetite. These actions of GLP-1, especially augmenting insulin secretion, may make the GLP-1R agonists beneficial in the treatment of T2DM. Although the GLP-1R agonists liraglutide (7) and semaglutide (8) have been shown to reduced cardiovascular outcomes in T2DM, exenatide has not.

There are some major limitations when considering using GLP-1R agonists in T1DM. Firstly, with the pancreatic $\beta$-cells are failing, as in the progression to T1DM, the GLP-1R agonists may be able to increase insulin secretion, but when they stop functioning, as in established T1DM, the GLP-1R agonists cannot increase insulin secretion. Secondly, although suppression of glucagon secretion is a physiologic response to GLP-1, it is not a consistent finding that the GLP-1R agonists suppress glucagon secretion in T1DM (9).

The MAG1C clinical trial (Meal-time Administration of Exenatide for Glycaemic Control in Type 1 Diabetic Cases) was started in December 2016 and completed in June 2019 (10). Prior to the start of MAG1C, only small benefits had 
been shown by adding short-acting exenatide to insulin in T1DM in the two studies undertaken. The first trial was an open-label crossover study of a small number of subjects (20 subjects) with long-standing T1DM, 17 of the subjects had some remaining pancreatic $\beta$-cell function and the potential to secrete insulin. In these subjects, treatment with exenatide for four months led to a reduction in body weight $(4.1 \mathrm{~kg})$ and decreased the use of injected insulin without increasing $\beta$-cell function/insulin secretion. In this study, exenatide also delayed gastric emptying, but had no effect on fasting glucagon levels. This combination of effects with exenatide led to a maintenance of glucose control with an HbA1c of $6.5 \%$ (11). The second trial was an open-label study in 18 subjects with new-onset T1DM and investigated the effect of exenatide, sitagliptin, or no addition to insulin on $\beta$-cell function. This was a pre- post- study, and after one year, it showed that exenatide added to injected insulin in six of the subjects, prevented insulin-induced weight gain and reduced the daily dose of insulin, without altering insulin secretion/ $\beta$-cell function. However, the reduction in HbA1c was the same in the insulin-treated group and the insulin plus exenatide group. In this trial, exenatide did not change the incidence of severe hypoglycaemia and there was no ketosis (12).

MAG1C was a randomised, double-blind, placebocontrolled clinical trial of mealtime exenatide in T1DM subjects taking insulin. Eighty-five percent of the funding came from AstraZeneca, the producer of the short-acting exenatide. To be enrolled subjects also had to have a BMI $>22 \mathrm{~kg} / \mathrm{m}^{2}$ and HbA1c in the range $7.5-10 \%$. Exclusions included hypoglycemic unawareness, diabetic gastroparesis and impaired kidney function. Many of the assessed subjects (498 of 654) declined the invitation or did not answer, leaving only a small number of participants (108 participants), and three of these were excluded from analysis as they probably had T2DM. The participants had a mean age of $\sim 50$ years, HbA1c of $\sim 8.3 \%$ and BMI of $\sim 28.3 \mathrm{~kg} / \mathrm{m}^{2}$. Participants were randomized to short-acting exenatide (5 $\mu \mathrm{g}$ escalated to $10 \mu \mathrm{g}$ after 2 weeks, subcutaneously) or placebo before breakfast, lunch and dinner. Side effects caused 10 of the subjects taking exenatide to discontinue its use, compared to none in the placebo group (13).

The primary endpoint of MAG1C was the betweengroup differences in HbA1c levels, and at 26 weeks, this was small at $-0.1 \%$ between the exenatide and placebo group, and not significantly different. Secondary endpoints included measures related to glucose levels (e.g., fasting glucose, postprandial glycemic excursions, time in hyperglycemia) and showed no difference between groups at 26 weeks. Exenatide significantly reduced body weight by $4.4 \mathrm{~kg}$, and total insulin requirements by 9.0 units/day. This reduction in insulin was due to reductions in prandial insulin dose, with no change in baseline insulin doses. Exenatide treatment had no effect on glucagon levels. The incidence of gastrointestinal adverse effects (nausea, vomiting, and acid reflux or heartburn) was higher with exenatide than placebo (13).

The authors concluded from MAG1C that short-acting exenatide did not seem to have a future as a standard addon treatment to insulin therapy in T1DM, mainly because it did not decrease HbA1c (13). It seems to me, that the GLP-1R agonists had no future in T1DM, could have been a possible conclusion before and during the MAG1C trial (9). Thus, prior to MAG1C, it was known that despite exenatide reducing body weight and the dose of insulin required in T1DM, but there was no evidence that this improved glucose control, and the results of MAG1C do not change these findings. Also, prior to MAG1C, there was no conclusive evidence that exenatide suppressed glucagon levels, and this was confirmed in MAG1C. Probably, the most significant new finding of MAG1C was that the gastrointestinal adverse effects of exenatide led to high levels of discontinuation.

Does the finding of no future with exenatide extend to other GLP-1R agonists? Probably. The GLP-1R agonist liraglutide has also been studied in T1DM. Small clinical trials of liraglutide in obese subjects with T1DM have shown reductions in HbA1c and body weight $(14,15)$. However, in larger phase 3 clinical trials, ADJUNCT ONE and ADJUNCT TWO, in addition to decreasing HbA1c and body weight, liraglutide also increased symptomatic hypoglycaemia and hypoglycemia with ketosis $(16,17)$. Due to these findings, Novo Nordisk decided not to apply to expand the use of liraglutide to T1DM (9). Other GLP-1R agonists include albiglutide, dulaglutide and semaglutide. Albiglutide has been compared to placebo in a phase 2 trial of 89 subjects with newly diagnosed T1DM. After 52 weeks of treatment with albiglutide, there was no change in body weight, insulin use, or HbA1c, but there was an excess of gastrointestinal adverse effects (18). Although a small trial has shown that dulaglutide decreased HbAlc and BMI in T1DM, it also increased gastrointestinal adverse effects (19). Thus, albiglutide and dulaglutide are also GLP-1R agonists not likely to be useful in T1DM. To my knowledge, the GLP-1R agonist semaglutide has not been tested in T1DM to date and given the disappointing results with the other 
GLP-1R, such testing seems inappropriate. In conclusion, in T1DM, exenatide and other GLP-1R agonists may improve some aspects of glycaemic control (e.g., HbA1c and body weight), but they may also have detrimental effects on other aspects of glycaemic control (hypoglycaemia, hyperglycemia with ketosis), and they all have adverse effects on the gastrointestinal tract. Consequently, GLP$1 \mathrm{R}$ agonists should probably no longer be considered for T1DM.

\section{Acknowledgments}

Funding: None.

\section{Footnote}

Provenance and Peer Review: This article was commissioned by the editorial office, Annals of Translational Medicine. The article did not undergo external peer review.

Conflicts of Interest: The author has completed the ICMJE uniform disclosure form (available at http://dx.doi. org/10.21037/atm-20-3702). The author has no conflicts of interest to declare.

Ethical Statement: The author is accountable for all aspects of the work in ensuring that questions related to the accuracy or integrity of any part of the work are appropriately investigated and resolved.

Open Access Statement: This is an Open Access article distributed in accordance with the Creative Commons Attribution-NonCommercial-NoDerivs 4.0 International License (CC BY-NC-ND 4.0), which permits the noncommercial replication and distribution of the article with the strict proviso that no changes or edits are made and the original work is properly cited (including links to both the formal publication through the relevant DOI and the license). See: https://creativecommons.org/licenses/by-nc-nd/4.0/.

\section{References}

1. Hughes DS, Narendran P. Alpha cell function in type 1 diabetes. Br J Diabetes Vasc Dis 2014;45-51.

2. de Ferranti SD, de Boer IH, Fonseca V, et al. Type 1 diabetes mellitus and cardiovascular disease: a scientific statement from the American Heart Association and American Diabetes Association. Circulation
2014;130:1110-30.

3. Liu Y, Song Y, Tao L, et al. Prevalence of diabetic retinopathy among 13473 patients with diabetes mellitus in China: a cross-sectional epidemiological survey in six provinces. BMJ Open 2017;7:e013199.

4. Barrett EJ, Liu Z, Khamaisi M, et al. Diabetic Microvascular Disease: An Endocrine Society Scientific Statement. J Clin Endocrinol Metab 2017;102:4343-410.

5. Frier BM. Hypoglycaemia in diabetes mellitus: epidemiology and clinical implications. Nat Rev Endocrinol 2014;10:711-22.

6. Conway B, Miller RG, Costacou T, et al. Temporal patterns in overweight and obesity in Type 1 diabetes. Diabet Med 2010;27:398-404.

7. Marso SP, Daniels GH, Brown-Frandsen K, et al. Liraglutide and Cardiovascular Outcomes in Type 2 Diabetes. N Engl J Med 2016;375:311-22.

8. Marso SP, Bain SC, Consoli A, et al. Semaglutide and Cardiovascular Outcomes in Patients with Type 2 Diabetes. N Engl J Med 2016;375:1834-44.

9. Doggrell SA. Do glucagon-like peptide-1 receptor (GLP-1R) agonists have potential as adjuncts in the treatment of type 1 diabetes? Expert Opin Pharmacother 2018;19:1655-61.

10. Meal-time administration of exenatide for glycaemic control in type 1 diabetic cases (MAG1C) ClinicalTrials. gov. Accessed 8th April, 2020. Available online: https:// clinicaltrials.gov/ct2/show/NCT03017352?term=exenatid e\&cond $=$ Type $+1+$ Diabetes $\&$ draw $=2 \&$ rank $=10$

11. Rother KI, Spain LM, Wesley RA, et al. Effects of exenatide alone and in combination with daclizumab on beta-cell function in long-standing type 1 diabetes. Diabetes Care 2009;32:2251-7.

12. Hari Kumar KV, Shaikh A, Prusty P. Addition of exenatide or sitagliptin to insulin in new onset type 1 diabetes: a randomized, open label study. Diabetes Res Clin Pract 2013;100:e55-8.

13. Johansen NJ, Dejgaard TF, Lund A, et al. Efficacy and safety of meal-time administration of short-acting exenatide for glycaemic control in type 1 diabetes (MAG1C): a randomised, double-blind, placebo-controlled trial. Lancet Diabetes Endocrinol 2020;8:313-24.

14. Kuhadiya ND, Malik R, Bellini NJ, et al. Liraglutide as additional treatment to insulin in obese patients with type 1 diabetes mellitus. Endocr Pract 2013;19:963-7.

15. Ghanim H, Batra M, Green K, et al. Liraglutide treatment in overweight and obese patients with type 1 diabetes: A 26-week randomized controlled trial; mechanisms of 


\section{Page 4 of 4}

weight loss. Diabetes Obes Metab 2020;22:1742-52.

16. Mathieu C, Zinman B, Hemmingsson JU, et al. Efficacy and Safety of Liraglutide Added to Insulin Treatment in Type 1 Diabetes: The ADJUNCT ONE Treat-To-Target Randomized Trial. Diabetes Care 2016;39:1702-10.

17. Ahrén B, Hirsch IB, Pieber TR, et al. Efficacy and Safety of Liraglutide Added to Capped Insulin Treatment in Subjects With Type 1 Diabetes: The ADJUNCT TWO
Doggrell. Exenatide at mealtimes in type 1 diabetes-no MAG1C here

Randomized Trial. Diabetes Care 2016;39:1693-701.

18. Pozzilli P, Bosi E, Cirkel D, et al. Randomized 52-week Phase 2 Trial of Albiglutide Versus Placebo in Adult Patients With Newly Diagnosed Type 1 Diabetes. J Clin Endocrinol Metab 2020;105:dgaa149.

19. Kutoh E, Hayashi J, Kuto AN. Efficacy and safety of dulaglutide in patients with absolute insulin deficiency. Endocr Regul 2019;53:187-90.
Cite this article as: Doggrell SA. Exenatide at mealtimes in type 1 diabetes-no MAG1C with exenatide, or with other glucagon-like peptide-1 receptor agonists. Ann Transl Med 2020;8(23):1610. doi: 10.21037/atm-20-3702 